\title{
Endoscopic biopsy of intra- and paraventricular brain tumors
}

\author{
Krzysztof Stachura, Ewelina Grzywna, Roger M. Krzyżewski, Borys M. Kwinta, Dariusz Adamek, Marek M. Moskała \\ Department of Neurosurgery and Neurotraumatology, Jagiellonian University Medical College, Krakow, Poland \\ Videosurgery Miniinv 2019; 14 (1): 107-113 \\ DOI: https://doi.org/10.5114/wiitm.2018.76117
}

\begin{abstract}
Introduction: Selection of the optimal treatment method of intra- and paraventricular tumors often requires histopathological verification that can be obtained by endoscopic biopsy.

Aim: To discuss the usefulness of the method in their own experience.

Material and methods: The results of 32 biopsies carried out during a 15-year period were reviewed retrospectively. All tumors were located supratentorially, 25 of them were intraventricular and 7 paraventricular. In 18 patients the tumor was accompanied by internal hydrocephalus. If the ventricular system was narrow, the biopsy was supported by a neuronavigation system. A rigid neuroendoscope was used. The obtained material was subjected to intraoperative and final histopathological examination.

Results: Viable diagnostic material was obtained from all patients. In 11 patients with tumor of the posterior portion of the third ventricle, cerebrospinal fluid was collected additionally for diagnostic tests. In 9 patients with obstructive hydrocephalus concomitant third ventriculostomy was performed. In 4 patients with tumor of the interventricular foramen, the tumor mass was reduced and in 2 cases septostomy was performed. In 3 (9.4\%) cases the histopathological diagnosis was descriptive and did not explain the nature of the lesion. Four biopsies resulted in persistent bleeding, in 3 patients transient memory impairments were observed, and in 1 patient an epileptic seizure occurred. Five patients needed ventriculoperitoneal shunt placement.

Conclusions: Endoscopic biopsy is a safe method to verify the histopathological nature of intra- and paraventricular lesions. It enables sampling of cerebrospinal fluid, reduction of tumor size, and in cases of coexisting obstructive hydrocephalus also third ventriculostomy or septostomy.
\end{abstract}

Key words: neuroendoscopy, intra- and paraventricular tumors, endoscopic biopsy.

\section{Introduction}

Brain tumors located intra- and paraventricularly, especially in the third ventricle and its proximity, remain a diagnostic difficulty due to their accessibility and diversity. Their histopathological verification is often necessary to make the right therapeutic decision. The advances in neuroendoscopic methods have contributed to application of endoscopic biopsy in this matter [1-7]. Neuroendoscopic biopsy seems to be, for many reasons, more advantageous than conventional stereotactic biopsy or open biop- sy. Currently many papers discuss the value of endoscopic biopsy in the treatment of intra- and paraventricular tumors [8-10]. However, due to the fact that intra- and paraventricular tumors represent about $2 \%$ of the primary central nervous system tumors, the presented material is small and often incoherent [11].

\section{Aim}

Authors, based on their many years of experience in performing endoscopic biopsy of intra- and

\footnotetext{
Address for correspondence

Krzysztof Stachura MD, PhD, Department of Neurosurgery and Neurotraumatology, Jagiellonian University Medical College,

3 Botaniczna St, 31-503 Krakow, Poland, phone: +48 1242486 60, e-mail: kkstach@poczta.onet.pl
} 
paraventricular brain tumors, demonstrate the efficacy and benefits of the method.

\section{Material and methods}

Over 15 years, 37 endoscopic biopsies of intraand paraventricular brain tumors were performed. All lesions were newly diagnosed based on the pre-hospital neuroimaging. The collected data were studied retrospectively and 5 patients with colloid cyst diagnosed intraoperatively (the cyst was removed after biopsy) were excluded. The study group consisted of 19 women and 13 men aged 19 to 74 years (mean age of 51 years). The most prevalent signs and symptoms reported by patients are presented in Table I. Six patients were asymptomatic and in these cases the brain tumor was an incidental finding. All head computed tomography (CT) and/ or magnetic resonance (MR) examinations met the criteria for three-dimensional imaging and were performed with contrast agent administration. All tumors were located supratentorially, 25 of them were intraventricular and 7 paraventricular. Table II presents their anatomical location. In 18 patients the tumor was accompanied by internal hydrocephalus.

Table I. Prevalent clinical signs and symptoms in 32 patients with the diagnosis of para- or intraventricular tumor

\begin{tabular}{|lc|}
\hline Signs and symptoms & No. of patients \\
\hline Headaches & 21 \\
\hline Transient impairments of consciousness & 10 \\
\hline Locomotive disorders & 6 \\
\hline Epilepsy & 3 \\
\hline Visual impairments & 4 \\
\hline Hypothalamic-pituitary failure & 2 \\
\hline Parinaud syndrome & 2 \\
\hline
\end{tabular}

Table II. Location of intra- and paraventricular tumors

\begin{tabular}{|lc|}
\hline Location & No. of patients \\
\hline Anterior portion of third ventricle & 15 \\
\hline Posterior portion of third ventricle & 11 \\
\hline Lateral ventricle & 6 \\
\hline Total & 32 \\
\hline
\end{tabular}

The diameters of the tumors ranged from $10 \mathrm{~mm}$ to $23 \mathrm{~mm}$. Precise analysis of neuroimaging studies included the meticulous assessment of tumor's consistency and vascularisation. In 6 cases computed tomography angiography was performed to exclude vascular malformation.

All patients were operated on under general anesthesia. The patient's position was determined by the location of the tumor. The head was positioned in the Mayfield clamp. In the case of the third ventricle tumor, the patient's head was bent to the chest. The endoscopic intraventricular approach led from the burr hole in the coronary area or anteriorly to the coronary area, $3 \mathrm{~cm}$ laterally from the sagittal suture on the right side, rarely left. In other cases, head positioning resulted from the chosen optimal surgical approach to the tumor that was determined by neuronavigation. Neuronavigation was also applied if the ventricular system was narrow and in some patients with tumors located in the posterior portion of the third ventricle. All procedures were performed using rigid Karl Storz neuroendoscopes: the GAAB model with Hopkins $0^{\circ}$ optics (having the outer diameter of $6.5 \mathrm{~mm}$ and $3 \mathrm{~mm}$ working channel) and the FRAZEE model with Hopkins $6^{\circ}$ optics (having the outer diameter of $8 \mathrm{~mm}$ and $5 \mathrm{~mm}$ working channel). The main advantage of the second one is the expanded toolkit. After cannulation of the ventricular system, and visualization and identification of the lesion, the biopsy was taken. Samples were collected using biopsy forceps, if possible from the surface and the intrinsic part of the tumor, 3-5 specimens in total. Coagulation was avoided before biopsy was completed. Bleeding was controlled by the irrigation of the operative field with warm Ringer's solution.

The collected specimens were sent to the neuropathology unit. One or two specimens were intended for intraoperative histopathological examination in which cytological methods were used - smear and/or cryosection with hematoxylin-eosin staining (HE staining). Remaining specimens were embedded in paraffin cubes, cut on the microtome and used for the final detailed histopathological verification of the tumor. Staining selection was adjusted individually, for case assessment and the need for differential diagnosis. In practice, in addition to HE staining, immunohistochemistry for GFAP, EMA, Ki67, LCA, cytokeratins and synaptophysin detection was done.

Each patient before discharge from hospital underwent a routine head CT scan. 
All procedures performed in studies involving human participants were in accordance with the ethical standards of the institutional and/or national research committee and with the 1964 Declaration of Helsinki and its later amendments or comparable ethical standards. For this type of study formal consent is not required.

\section{Results}

Viable diagnostic material was obtained from all patients. In 11 patients with a tumor of the posterior portion of the third ventricle, cerebrospinal fluid (CSF) was collected additionally for diagnostic tests before the biopsy was taken. In 9 of them with obstructive hydrocephalus, endoscopic third ventriculostomy (ETV) was performed prior to biopsy. In 4 patients with a tumor adjacent to the interventricular foramen, tumor mass reduction restored the CSF circulation. In the remaining 2 cases, septostomy was performed.

Histopathological findings are presented in Table III. In $3(9.4 \%)$ cases the histopathological diagnosis was descriptive and did not explain the nature of the lesion. Twice it was due to the limited amount of diagnostic material.

In 4 cases, the biopsy resulted in persistent bleeding, which required a several-minute infusion of warm Ringer's solution to control the hemorrhage. Preoperative imaging in these patients revealed rich tumor vascularization. The intraoperative bleeding did not result in surgery cessation in any case. However, in 3 cases, the lack of full hemostasis in the ventricular system led to external ventricular drainage (EVD) placement that was maintained for several days. Three patients presented transient memory impairments in the early postoperative period, resulting from probable fornix injury. One patient developed epileptic seizures that were managed with antiepileptic drugs. Five patients needed ventriculoperitoneal shunt placement due to persistent symptoms of hydrocephalus. There was no deterioration of pre-existing neurological deficits. There were no inflammatory complications. No deaths were reported. The routine head CT scan allowed exclusion of other reasons that could prevent discharge from hospital (Photos 1, 2).

\section{Discussion}

Para- and intraventricular brain tumors often manifest similar clinical and radiological symptoms, but they represent a very broad histopathological spectrum. Hence, the histopathological verification should determine the selection of treatment method.

The first endoscopic biopsy of an intraventricular tumor was performed by Fukushima and described in 1978 [12]. With development of neuroendoscopic instrumentation and with advances in techniques supporting neuroendoscopy, the endoscopic biopsy of ventricular system tumors is becoming more and more recognized. Its undoubted advantage is the selection of the biopsy site under direct vision. This allows one to avoid the parts with rich vascularization and obtain the material from the area already showing macroscopic features of pathology. The most important step in the procedure is proper planning of the procedure itself. It is recognized that any endoscopic procedure within the ventricular system beyond the interventricular foramen area should be assisted by the neuronavigation system [13-15]. Our own and others' experience demonstrates that it is also useful when performing biopsy in a narrow ventricular system [16-18]. When neuronavigation is in use, unnecessary CSF leakage should be avoided, since this may

Table III. Histopathological characteristics of biopsy material

\begin{tabular}{|lc|}
\hline Histopathological diagnosis & No. of patients \\
\hline Pilocytic astrocytoma & 6 \\
\hline Fibrillary astrocytoma & 4 \\
\hline Subependymoma & 4 \\
\hline Ependymoma & 3 \\
\hline Craniopharyngioma & 3 \\
\hline Glial cyst & 2 \\
\hline Anaplastic astrocytoma & 1 \\
\hline $\begin{array}{l}\text { Dysembryoplastic neuroepithelial tumor } \\
\text { (DNT) }\end{array}$ & 1 \\
\hline Ganglioglioma & 1 \\
\hline Pineoblastoma & 1 \\
\hline B-cell lymphoma & 1 \\
\hline Germinoma & 32 \\
\hline Metastasis & 1 \\
\hline Descriptive diagnosis & 3 \\
\hline Total & 1 \\
\hline
\end{tabular}



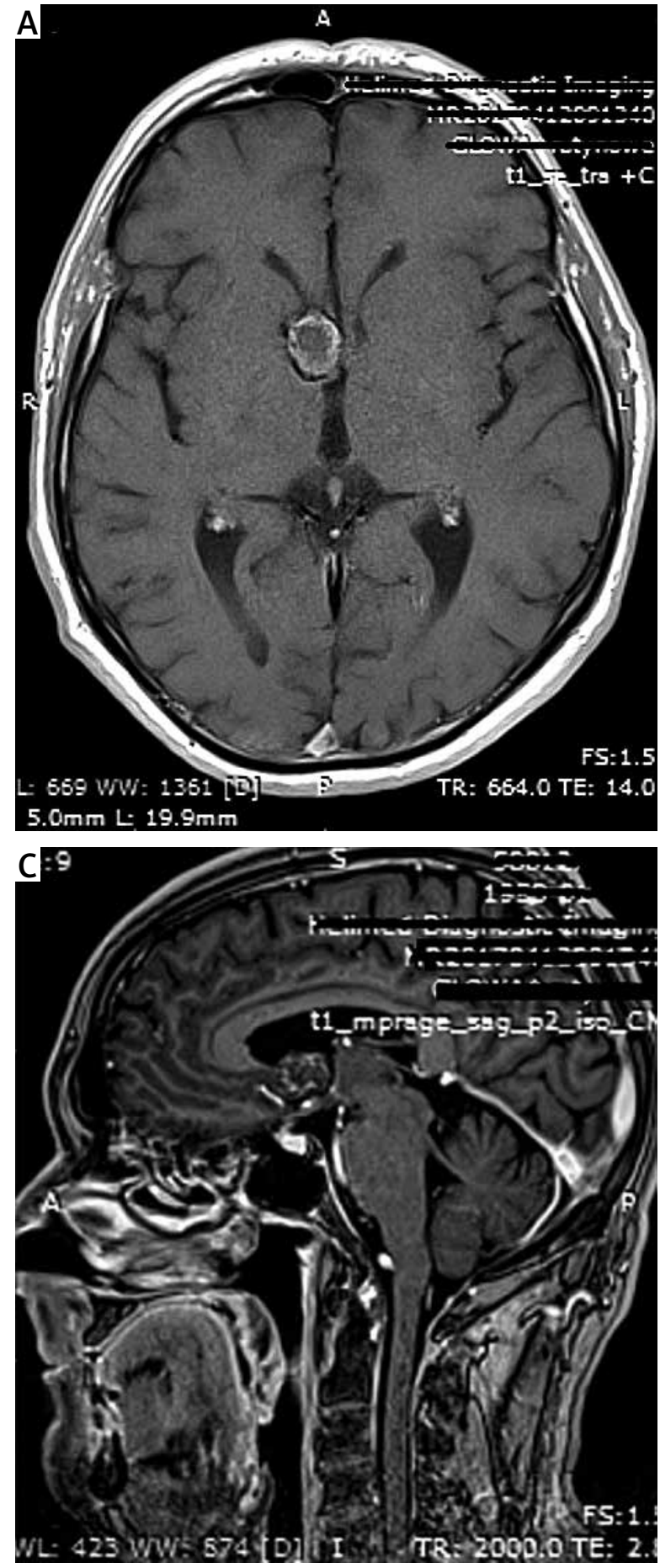

distort the transmission of real-time position of the used tools. Using a rigid endoscope guarantees that the planned trajectory to access the target is maintained. Moreover, it enables one to obtain CSF samples for neoplasm marker testing, which is of particular significance in the case of management

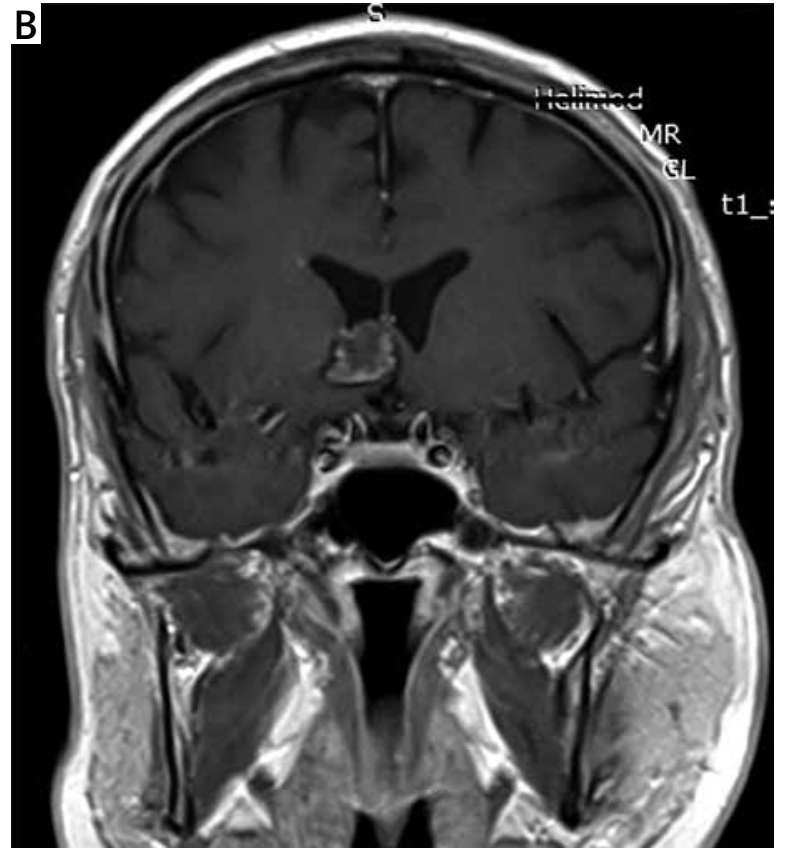

Photo 1. MR (A) axial, (B) coronal and (C) sagittal T1-weighted image. Paraventricular tumor in posterior portion of right lateral ventricle anterior horn

of tumors of the posterior portion of the third ventricle. In selected cases with co-existing obstructive hydrocephalus there is a possibility to perform concomitant ETV or septostomy prior to biopsy. Consequently, ventriculoperitoneal shunting threatening neoplasm dissemination is avoided. 

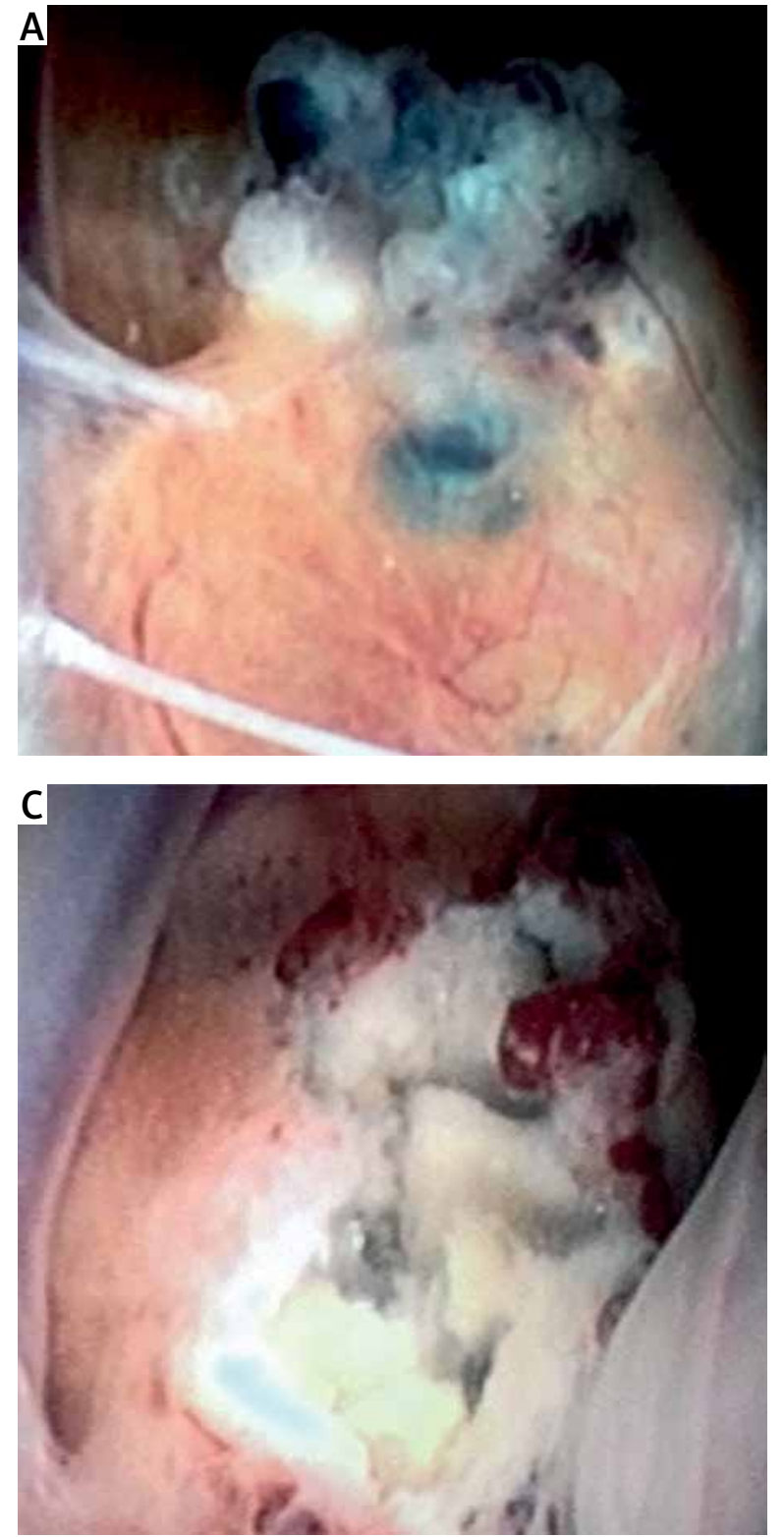

The feasibility of endoscopic biopsy in the diagnostics of intra- and paraventricular lesions is estimated at 69.9-100\% [1, 5, 7-9, 14, 19-25]. This vast range results from the wide heterogeneity of studied patient groups, the adopted methodology and experience of the research team. In the present material histopathological diagnosis was achieved in $90.6 \%$ of cases. In the largest material so far, concerning 691 patients, Hayashi et al. achieved a $94.7 \%$ rate of conclusive diagnosis [21]. Similar results are presented in other reports, including the multicenter studies conducted in Italy and the study by Giannetti et al. $[7,8]$. Histopathological verification does not always

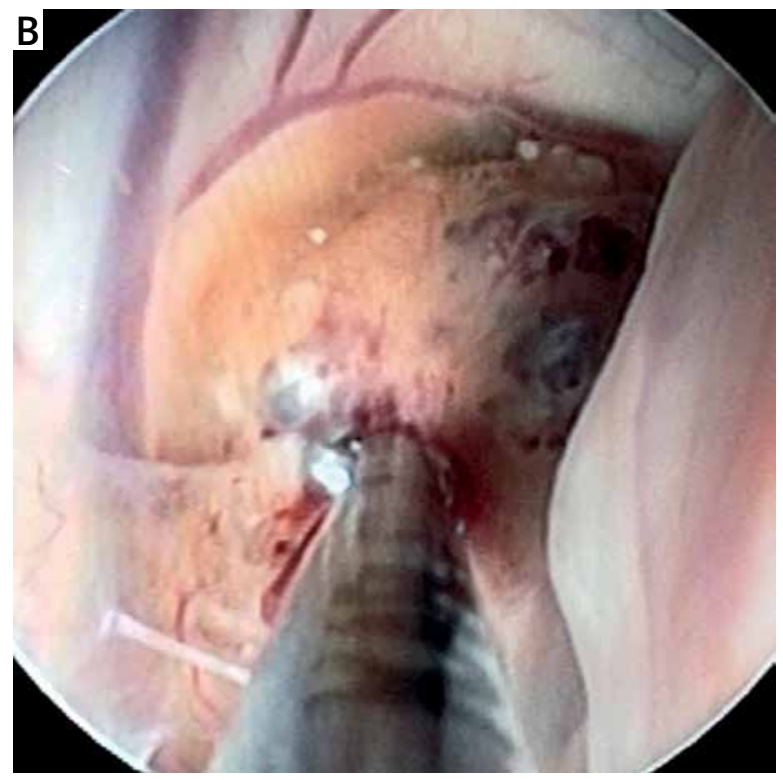

Photo 2. Endoscopic view. A - Paraventricular tumor in posterior portion of right lateral ventricle anterior horn, B - tumor biopsy, C - view after biopsy

determine the final clinical diagnosis - as in three of the presented cases. Depreitere et al. have classified the results of endoscopic biopsies of intraventricular tumors in four categories. The first category includes those histopathological diagnoses that have not raised any objections. In the second, the diagnosis raised minor objections, but with strong arguments in favor of the made diagnosis. In the third category were reports of abnormal or neoplastic tissues, but a final diagnosis was unlikely to be made, and the fourth category includes reports on specimens that did not contain abnormal tissue or on specimens impossible to interpret [19]. While the first two catego- 
ries have a diagnostic dimension, the other two do not. The most common causes of diagnostic difficulties are an insufficient amount of material collected during biopsy, inaccurate sampling place or use of coagulation before taking the biopsy. Some reports undermine the accuracy of the endoscopic biopsy. These assumptions are based on comparison of diagnosis achieved in biopsy and open surgery [7]. The accuracy of histopathological diagnoses in conventional biopsy is comparable [26].

Endoscopic biopsy carries relatively low risk of complications and low mortality. The most serious complication, as in other endoscopic procedures, is hemorrhage. The authors have experienced it in 4 cases and it was directly related to the biopsy itself. Assessment of bleeding intensity is most often subjective. In the paper of Giannetti et al. mentioned previously, bleeding was defined as relevant when EVD placement or additional surgical intervention was needed for closure. The potential risk of significant bleeding is estimated at 0-9.7\% [7]. Other noted complications were transient memory impairments and epileptic seizures that did not affect the patients' outcome. The incidence of inflammatory complications, reoperation or death resulting from endoscopic biopsy is estimated in the literature at 0-6.5\% [1, 5, 7-9, 14, 19-25].

\section{Conclusions}

Endoscopic biopsy is a safe, minimally invasive procedure that enables histopathological verification of intra- and paraventricular tumors and sampling of CSF for diagnostic tests. In cases with co-existing obstructive hydrocephalus, concomitant ETV, septostomy or tumor mass reduction during the endoscopic biopsy restores CSF circulation.

\section{Conflict of interest}

The authors declare no conflict of interest.

\section{References}

1. Gaab MR, Schroeder HW. Neuroendoscopic approach to intraventricular lesions. J Neurosurg 1998; 88: 496-505.

2. Souweidane MM, Sandberg DI, Bilsky MH, et al. Endoscopic biopsy for tumors of the third ventricle. Pediatr Neurosurg 2000; 33: 132-37.

3. Yurtseven T, Ersahin Y, Demirtas E, et al. Neuroendoscopic biopsy for intraventricular tumors. Minim Invasive Neurosurg 2003; 46: 293-9.
4. Luther N, Edgar MA, Dunkel IJ, et al. Correlation of endoscopic biopsy with tumor marker status in primary intracranial germ cell tumors. J Neurooncol 2006; 79: 45-50.

5. Mohanty A, Santosh V, Devi Bl, et al. Efficacy of simultaneous single-trajectory endoscopic tumor biopsy and endoscopic cerebrospinal fluid diversion procedures in intra- and paraventricular tumors. Neurosurg Focus 2011; 30: E4.

6. Morgenstern PF, Osbun N, Schwartz TH, et al. Pineal region tumors: an optimal approach for simultaneous endoscopic third ventriculostomy and biopsy. Neurosurg Focus 2011; 30: E3.

7. Giannetti AV, Holayama Alvarenga AY, Lemos de Lima TO, et al. Neuroendoscopic biopsy of brain lesions: accuracy and complications. J Neurosurg 2015; 122: 34-9.

8. Oppido PA, Fiorindi A, Benvenuti L, et al. Neuroendoscopic biopsy of ventricular tumors: a multicentric experience. Neurosurg Focus 2011; 30: E2.

9. Dominiguez-Paez M, Puch-Ramirez M, Rodriguez-Barcelo S, et al. Neuroendoscopic biopsy. Experience in 31 patients and literature review. Neurocirurgia (Astur) 2011; 22: 419-27.

10. Constatntini S, Mohanty A, Zymberg S, et al. Safety and diagnosis accuracy of neuroendoscopic biopsies: an international multicenter study. J Neurosurg Pediatr 2013; 11: 704-9.

11. Waldron JS, Tihan T. Epidemiology and pathology of intraventricular tumors. Neurosurg Clin N Am 2003; 14: 469-82.

12. Fukushima T. Endoscopic biopsy of intraventricular tumors with the use of the ventriculofiberoscope. Neurosurgery 1978; 2: 110-13.

13. Stachura K, Libionka W, Czepko R. The use of neuroendoscopy in the treatment of intraventricular or paraventricular brain tumors. Neurol Neurochir Pol 2005; 39: 101-7.

14. Hussain I, Abdel-Latif AM, Souweidane MM. Intraventricular and paraventricular tumors. In: Neuroendoscopic Surgery. Torres-Corzo JM, Rangel-Castilla L, Nakaji P (eds). Thieme. New York, Stuttgart, Delhi, Rio de Janeiro 2016; 127-43.

15. Roszkowski M. Neuronavigation - image guided surgery, current application in neurosurgery. Probl Lek 2006; 45: 17-26.

16. Cappabianca P, Cinalli G, Gangemi M, et al. Application of neuroendoscopy to intraventricular lesions. Neurosurgery 2008; 62 (Suppl 2): 575-98.

17. Stachura K, Grzywna E. Neuronavigation-guided endoscopy for intraventricular tumors in adult patients without hydrocephalus. Videosurgery Miniinv 2016; 11: 200-7.

18. Prat R, Galeano I. Endoscopic biopsy of foramen of Monro and third ventricle lesion guided by frameless neuronavigation: usefulness and limitation. Clin Neurol Neurosurg 2009; 111: 579-82.

19. Depreitere B, Dasi N, Rutka J, et al. Endoscopic biopsy for intraventricular tumors in children. J Neurosurg 2007; 106 (5 Suppl): 340-6.

20. Fiorindi A, Longatti P. A restricted neuroendoscopic approach for pathological diagnosis of intraventricular and paraventricular tumours. Acta Neurochir 2008; 150: 1235-9.

21. Hayashi N, Murai H, Ishihara S, et al. Nationwide investigation of the current status of therapeutic neuroendoscopy for ventricular and paraventricular tumors in Japan. J Neurosurg 2011; 115: 1143-57. 
22. Macarthur DC, Buxton N, Punt J, et al. The role of neuroendoscopy in the management of brain tumours. $\mathrm{Br} J$ Neurosurg 2002; 16: 465-70.

23. Pople IK, Athanasiou TC, Sandeman DR, et al. The role of endoscopic biopsy and third ventriculostomy in the management of pineal region tumours. Br J Neurosurg 2001; 15: 305-11.

24. Song JH, Kong DS, Shin HJ. Feasibility of neuroendoscopic biopsy of pediatric brain tumors. Childs Nerv Sys 2010; 26: 1593-8.

25. Ahn ES, Goumnerova L. Endoscopic biopsy of brain tumors in children: diagnosis success and utility in guiding treatment strategies. J Neurosurg Pediatr 2010; 5: 255-62.

26. Mennel HD, Hellwig D, Bauer BL. Results and reliability of stereotactic and endoscopic biopsies in brain tumors. Zentralb Neurochir 1994; 55: 79-90.

Received: 5.04.2018, accepted: 12.05.2018. 